\title{
Primary Care Clinicians' Beliefs and Strategies for Managing Chronic Pain in an Era of a National Opioid Epidemic
}

\author{
Laura G. Militello, $M A^{\top} \odot$, Robert W. Hurley, MD, PhD², Robert L. Cook, MD $D^{3,4}$, \\ Elizabeth C. Danielson, $M A^{5}$, Julie Diiulio, $M S^{7}$, Sarah M. Downs, MPH ${ }^{5}$, Shilo Anders, $P h D^{6}$, \\ and Christopher A. Harle, $P h D^{7}$
}

\begin{abstract}
${ }^{1}$ Applied Decision Science, LLC, 5335 Far Hills Ave, Suite 103, Dayton, OH, USA; ${ }^{2}$ Department of Anesthesiology and Public Health, Wake Forest University School of Medicine, Winston-Salem, NC, USA; ${ }^{3}$ Department of Epidemiology, University of Florida, Gainesville, FL, USA; ${ }^{4}$ Department of Medicine, University of Florida, Gainesville, FL, USA; ${ }^{5}$ Department of Health Policy and Management, Indiana University Richard M. Fairbanks School of Public Health, Indianapolis, IN, USA; ${ }^{6}$ Center for Research and Innovation in Systems Safety, Vanderbilt University Medical Center, Nashville, TN, USA; ${ }^{7}$ Department of Health Outcomes and Biomedical Informatics, University of Florida, Gainesville, FL, USA.
\end{abstract}

\begin{abstract}
BACKGROUND: Little is known about how primary care clinicians (PCCs) approach chronic pain management in the current climate of rapidly changing guidelines and the growing body of research about risks and benefits of opioid therapy.
\end{abstract}

OBJECTIVE: To better understand PCCs' approaches to managing patients with chronic pain and explore implications for technological and administrative interventions.

DESIGN: We conducted adapted critical decision method interviews with 20 PCCs. Each PCC participated in 1-5 interviews.

PARTICIPANTS: PCCs interviewed had a mean of 14 years of experience. They were sampled from 13 different clinics in rural, suburban, and urban health settings across the state of Indiana.

APPROACH: Interviews included discussion of participants' general approach to managing chronic pain, as well as in-depth discussion of specific patients with chronic pain. Interviews were audio recorded. Transcripts were analyzed thematically.

KEY RESULTS: PCCs reflected on strategies they use to encourage and motivate patients. We identified four associated strategic themes: (1) developing trust, (2) eliciting information from the patient, (3) diverting attention from pain to function, and (4) articulating realistic goals for the patient. In discussion of chronic pain management, PCCs often explained their beliefs about opioid therapy. Three themes emerged: (1) Opioid use tends to reduce function, (2) Opioids are often not effective for long-term pain treatment, and (3) Response to pain and opioids is highly variable.

CONCLUSIONS: PCC beliefs about opioid therapy generally align with the clinical evidence, but may have some important gaps. These findings suggest the potential value of interventions that include improved access to research findings; organizational changes to support PCCs

Electronic supplementary material The online version of this article (https://doi.org/10.1007/s11606-020-06178-2) contains supplementary material, which is available to authorized users.

Received February 21, 2020

Accepted August 24, 2020

Published online September 9, 2020 in spending time with patients to develop rapport and trust, elicit information about pain, and manage patient expectations; and the need for innovative clinical cognitive support.

KEY WORDS: chronic pain; opioid; primary care; pain management; qualitative research.

$\mathrm{J}$ Gen Intern Med 35(12):3542-8

DOI: $10.1007 / \mathrm{s} 11606-020-06178-2$

(c) Society of General Internal Medicine 2020

\section{INTRODUCTION}

When it comes to pain management, primary care clinicians (PCCs) find themselves in a somewhat unexpected role. (1) Few conditions intersect with a range of specialties (i.e., mental health, orthopedics, endocrinology, etc.), disability, and aberrant behavior in the way that chronic pain does. PCCs find themselves in a position where they are asked to assess and diagnose sometimes vague and diffuse pain, and determine appropriate treatment often before the underlying cause of the pain is well-understood. A recent cultural shift in the USA has created a situation in which a formerly default treatment, prescription opioid therapy, is no longer considered safe or appropriate for many patients with chronic pain. The addictive qualities and overall safety profile of opioid medications have come into sharp focus in recent years, leading to a push to reduce opioid use while also trying to achieve pain relief with little guidance for PCCs about how to manage this change in treatment plans. $(2,3)$ Others have documented the uneasiness many experience in managing patients with chronic pain $(4,5)$. One participant in our study described the sense that opioid prescribing sometimes extends into unexpected and disconcerting territory in this way: "I never signed up to be an enforcer." The complexity and moral uncertainty (6) associated with managing patients with chronic pain are an important backdrop for the findings from this study.

Despite limited formal training about chronic pain $(7,8)$, PCCs play a critical role in supporting patients as they seek to optimize quality of life while managing the risks associated 
with different treatments. The current study extends prior studies highlighting the emotional burden, inadequate resources, lack of trust between patient and provider (4), and patient-provider communication challenges (5) associated with managing patients with chronic pain. Much prior research was conducted using surveys and focus groups. To extend this body of knowledge, we conducted in-depth interviews with individual PCCs in the context of the recently released CDC guidelines which prompted many institutions to adopt strict opioid prescribing guidelines. (9) Our intent was to better understand current beliefs and strategies for managing noncancer chronic pain in this new era.

\section{METHODS}

\section{Overall Study Design}

In this study, we asked PCCs to describe their general approach to managing patients with chronic pain, and then to walk us through examples of such patients so that we could understand how this general approach is applied and tailored in the context of real patients. The resulting context-rich dataset provides insights not easily obtained with surveys or observational studies. In this paper, we focus on the portions of the interviews in which PCCs shared their evolving understanding of opioid therapy, and the strategies they have developed to encourage and motivate patients.

\section{Participants}

From April 2016 to July 2018, we recruited a purposive sample of 20 PCCs who prescribe opioids and treat patients with chronic noncancer pain. Recruiting strategies included email invitations, in-person presentations at clinic staff meetings, and word-of-mouth. Participants were given a $\$ 100$ gift card for each interview. Our sample included PCCs from three health systems, including one non-profit hospital system with primarily rural clinics in Indiana and Illinois; a safety net hospital serving a major urban center in Indiana; and an academic medical center with hospitals and clinics throughout Indiana in rural, urban, and suburban regions. Our initial plan was that each PCC would participate in up to 5 interviews. We analyzed transcript as they became available, determining we had reached thematic saturation (i.e., when additional data did not reveal any new themes) (10-12) after 89 interviews with 20 providers.

\section{Data Collection and Analysis}

The initial interview with each PCC began with questions about the PCC's patient population; general approach to chronic noncancer pain care; and tools and instruments used when caring for patients with chronic noncancer pain. The remainder of the initial interview and all subsequent interviews with each PCC used an adapted critical decision method technique (4), focusing on a recent patient that the PCC confirmed to have a chronic noncancer musculoskeletal pain condition. A different patient was discussed in each interview session. Follow-up probes were used to explore cues, goal, strategies, and actions taken for key events in the patient's history. Traditional critical decision method interviews focus on eliciting a challenging incident from the perspective of the interviewee. The interviewee identifies a challenging incident from memory; the interviewer elicits a timeline of the incident, and then applies cognitive probes and hypotheticals to explore assessments, goals, and strategies. For this study, we adapted the method to focus on a recent patient encounter so that we could request permission from the patient to access their electronic health record during the interview as a memory support. This interview technique was selected because of its strength in obtaining rich detail and increased accuracy over more general interviews. $(13,14)$

Interviews were conducted with individual PCCs by $1-3$ interviewers, lasting approximately $60 \mathrm{~min}$. After the first 15 interviews, we conducted preliminary analysis and refined the interview guide to include questions about opioid-related risks, benefits, and goals. In order to accommodate clinician time constraints and increase standardization, we also shifted to a more streamlined interview approach, adhering more closely to the interview guide and restricting follow-up questions to clarifications (see ESM 1) for initial interview guide with refinements highlighted).

We conducted a thematic analysis on deidentified interview transcripts, examining both abstract descriptions of each participant's general approach to managing patients with chronic pain and specific examples. $(15,16)$ To develop a codebook, each team member (four behavioral researchers, two primary care clinicians, one pain specialist physician) reviewed two transcripts and identified potential coding categories that would support the team in articulating themes. Potential coding categories were influenced by the curiosity of the research team, the overall study goal of identifying leverage points for supporting PCCs in managing patients with chronic pain, and the data itself. Two of the behavioral researchers were health services researchers and two were cognitive engineers. Four behavioral researchers compiled responses and applied the resulting draft codebook to three interview transcripts using Dedoose version 8.1.10. The analysis team met after each to discuss and refine until consensus was reached about the codebook. Two behavioral researchers then coded remaining interview transcripts as they became available, meeting after each to reach consensus on all codes. Behavioral researchers discussed recurring themes and new ideas throughout the process, determining that thematic saturation had been reached when no new themes emerged after analyzing transcripts from several consecutive participants. (10-12) 


\section{FINDINGS}

\section{Clinician Characteristics}

Our sample included 20 PCCs, who represented 13 clinics across three not-for-profit Indiana healthcare systems (Table 1).

\section{Strategies to Encourage and Motivate Patients}

PCCs described deliberate strategies they find effective in managing patients with chronic pain. Many emphasized that chronic pain influences all aspects of a patient's life. Thus, the PCC is often in a role of encouraging and motivating patients to adhere to treatment recommendations, to make lifestyle changes, to accept limitations, and to celebrate progress. We identified four themes related to encouraging and motivating patients: (1) developing trust, (2) eliciting information from the patient, (3) diverting attention from pain to function, and (4) articulating realistic goals for the patient.

Developing Trust. Some PCCs emphasized deliberate strategies they use to develop trust with patients. One described active listening: "I'm listening, which is a very important thing I think in connecting with a human being is to listen to them. When I am asking questions, ... I am actually really trying to say ... I am interested in what you are concerned about. The questions create this bridge where there is trust, so they know I care and trust me."

Some highlighted how much can be learned by letting the patient talk, particularly for patient populations that find it difficult to ask for help:

... letting him talk, getting him to ramble. I mean, listen to him about his cars and all that stuff, ..., but you get them to where they are comfortable and then over a period of time ... they spill their guts and you know what's going on. Because, especially middle-aged rural men, they aren't going to tell you. I mean, 'you can ask me all kinds of things that I know are important, that you may have a legitimate need to know, but I'm not going to tell you. I'm going to manage ... I take care of my own problems,' and so that's the mentality, so you've got to sneak it, you've got to get it out of them whenever you can...

Eliciting Information from the Patient. PCCs emphasized the importance of eliciting information from the patient to inform the pain management plan. One PCC explained: "Yeah, I just try to ask more and more specific questions you know to try to get as objective of responses as I can to you know kind of standard questions, rather than [accepting the response:] 'oh this is like horrible.' So, say: 'what's horrible about it, show me where does it hurt, show me exactly where, you know?' Stuff like that."

Another PCC characterized eliciting patient motivations as mining for gold: "And if they haven't developed a motivation, I kind of think about talking to a patient is like mining for gold, you have to sift through a lot of dirt to get the little gold nuggets. So, trying to help them identify that and creating tiny little gold."

Diverting Attention from Pain to Function. PCCs explained how some patients become captive by their pain: "The twilight zone, you know, like you can't escape." To address this, some PCCs described deliberate strategies to shift the focus from pain to function using a "small steps" strategy. One described asking patients: "What kind of activities are you able to get done with your pain the way it is right now?" and recording the response every time so the PCC could highlight improvements over time. This was a shift from that PCC's previous pain-focused approach that included asking patients

Table 1 Participant Characteristics

\begin{tabular}{|c|c|c|c|c|c|c|}
\hline & Gender & Degree & Specialty & Practice setting & Years in practice & No. of interviews \\
\hline Provider 1 & Female & MD & Internal medicine & Urban & 5 & 5 \\
\hline Provider 2 & Male & $\mathrm{MD}$ & General medicine & Urban & 26 & 5 \\
\hline Provider 3 & Female & MD & Internal medicine & Urban & 17 & 5 \\
\hline Provider 4 & Female & MD & Family medicine & Urban & 12 & 5 \\
\hline Provider 5 & Female & FNP & Family medicine & Urban & 15 & 5 \\
\hline Provider 6 & Male & $\mathrm{DO}$ & Family medicine & Rural & 30 & 4 \\
\hline Provider 7 & Male & MD & Family medicine & Rural & 2.5 & 5 \\
\hline Provider 8 & Female & MD & Family medicine & Rural & 2 & 5 \\
\hline Provider 9 & Male & MD, FAAFP & Family medicine & Urban & 16 & 5 \\
\hline Provider 10 & Female & $\mathrm{MD}$ & Internal medicine & Urban & 16 & 5 \\
\hline Provider 11 & Male & MD, FAAFP & Family medicine & Urban & 11 & 5 \\
\hline Provider 12 & Male & $\mathrm{DO}$ & Family medicine & Urban & 4 & 5 \\
\hline Provider 13 & Male & MD & Family medicine & Urban & 23 & 5 \\
\hline Provider 14 & Female & $\mathrm{MD}$ & Family medicine & Suburban & 4 & 5 \\
\hline Provider 15 & Female & MD & Med-Peds & Urban & 7.5 & 5 \\
\hline Provider 16 & Male & MD & Med-Peds & Urban & 26 & 5 \\
\hline Provider 17 & Male & MD & General medicine & Urban & 19 & 1 \\
\hline Provider 18 & Female & MD & Internal medicine & Urban & 10 & 5 \\
\hline Provider 19 & Female & NP & Adult medicine & Urban & 2.5 & 2 \\
\hline Provider 20 & Male & MD & Family medicine & Rural & 34 & 2 \\
\hline
\end{tabular}


to rate pain on a scale of 1 to 10 during each visit. A common emphasis is understanding how pain limits the patient's life and what s/he would like to be able to do that s/he cannot. One PCC described the conversation this way "... okay, we want to improve your functioning, and what does that mean for you? Does that mean being able to go to church? Or being able to go to the park with your grandkids..."

Articulating Realistic Goals for the Patient. Efforts to learn more about the patient's limitations and goals support the PCC's ability to articulate realistic goals for the patient. A common theme among PCCs is the effort to help patients understand that their pain is not going to go away completely: "I try to make sure that the patient understands what realistic goals for care are, that we're probably not going to make the pain go away, but at least we can make them more functional." One PCC reported that they take a more directive approach when managing chronic pain: "...I used to have the patients set the goals more. That is a change. That's a big change from what I used to do five years ago, for example.... I definitely do a lot more setting the goals, but you know explaining why."

A discussion about goals often involves explaining to patients that opioids may mask pain, but they do not actually treat the problem; therefore, reducing or limiting use of opioids is an important goal. Some indicated that this conversation has become easier as the risks of opioid therapy have been highlighted in the news in recent years, setting the stage for a discussion of realistic goals: It wasn't just in the medical literature that there was a problem, it was in lay press that there were problems, so the environment changed. And because of that, it made it easier to say to patients, well yeah, I know you've got pain, but I don't think these pain medicines, these narcotics are what you need, or I sure don't think a bunch of it is what you need, maybe limited amounts but no we're just not going to keep going up and up on the doses.

\section{Beliefs About Opioid Therapy}

In response to questions about their approach to managing patients with chronic pain, PCCs often explained their beliefs about opioid therapy. We use the term "belief" to refer to statements describing PCC's understanding of opioids and their effects on patients with chronic pain. PCCs described these beliefs as evolving insights and offered underlying rationale that informed patient interactions and treatment approaches. Three themes emerged from these data: (1) Opioid use tends to reduce function, (2) Opioids are often not effective for long-term pain treatment, and (3) Response to opioids is highly variable.

Opioid Use Tends to Reduce Function. Many PCCs explained that opioids reduce patients' ability to function in other aspects of their lives. Some noted that people on opioids tend to neglect other aspects of health: "One of the big concerns in my mind and in a lot of people's minds now is that people on chronic opioids - what is clear just by observing these people - is that they neglect a lot of their other health... and I can't give you any published data on that." Others reported observable improvements in other health measures when patients are weaned from opioids. Some reported that cognitive function improves when people stop using opioids: I've seen patients who get off opioids and benzodiazepines and say: "I can finally think"... and their cognitive functioning improves. One PCC framed this issue in terms of motivation: "That tool [opioids] helps kind of taking away that, you know, that drive...." Many PCCs recalled first-hand experiences with patients and anecdotes that influenced their beliefs about the effect of opioid use on function.

Opioids Are Often Not Effective for Long-term Pain Treatment. Many PCCs reported that opioids are not effective for treating chronic pain over time. Some frame this in terms of building up a tolerance to the medication: "Because of the tolerance that people build up to opioids, they don't really work well in the majority of patients." One went further, indicating that not only are opioids less effective over time, but they can exacerbate pain symptoms: "the longer somebody is on [opioid] pain medicine, the more pain they actually feel."

Response to Pain and Opioids Is Highly Variable. PCCs described individual differences in patients they observed. For example, one hypothesized that athletes are better at managing pain in general: "I see people who run, who are marathoners, who are bikers, who continue to work out and know what good pain is and they seem to tolerate things better.... I don't know if our brain sets up something differently when we learn what good pain is and we expect a little bit of discomfort..." Another PCC explained that the analgesic effect is not completely predictable: "There are certain people that take limited amounts of opioids on a regular basis, and they seem to do OK, but a lot of people don't even find them all that useful over time." As a result, it is hard to know who will experience pain relief with a specific medication. It is also difficult to determine who will become addicted, and who will become less functional.

See ESM 2 for additional quotes to support these themes.

\section{DISCUSSION}

PCC reported beliefs about opioid risks and efficacy generally align with the evidence base. (16) Many PCCs reported a belief that long-term opioid therapy (LTOT) is often not effective for pain relief, although no one in this sample highlighted relevant research findings. $(17,18)$ Similarly, PCCs generally discussed the link between reduced function and opioid use as an insight they and their colleagues had 
noted over time rather than an evidence-based finding. (19) One point of controversy is that many report that LTOT can increase pain; yet, there is limited evidence of opioid-induced hyperanalgesia. (20) It is not surprising that observing patients' inability to manage other chronic conditions while using opioids (or the increase in function after stopping opioids) combined with limited pain relief would be salient to a clinician and reinforce the risks of opioid therapy in a very personal way.

The frequent mention of anecdotes and observed trends as evidence suggests that many of the clinicians in this sample have reflected on and are actively seeking to understand chronic pain and how to most effectively manage patients who experience chronic pain. This is no doubt amplified by the fact that research findings about the effects of LTOT are confusing and contradictory at times. For example, although there is clear risk associated with LTOT, stopping opioids may increase risk in some cases (21), and opioid tapering may exacerbate pain. $(22,23)$

In discussion of strategies to motivate and encourage patients, it is notable that many PCCs in our sample described their approach as unconventional. Deliberately making time to develop trust in the context of chronic pain involves having the skills to elicit and listen for indirect cues, flawed beliefs, and goals that may motivate the patient. Prior studies indicating that many patients with chronic pain report feeling distrusted and that their symptoms and concerns were dismissed as trivial in interactions with the PCCs and specialists $(6,8,24)$ align with our participants' perception that these effective, but timeconsuming, strategies to build trust are not universal. Many described a shift in approach as they have come to understand chronic pain more fully. Remarkably, PCCs in this study found ways to develop relationships and facilitate discussion of risks and goals, in spite of the well-documented time constraints in primary care. $(25,26)$ Future research into LTOT use should be informed by these insightful experiences from the front lines.

One implication of these findings is that much of the knowledge and insight that drives chronic pain management is currently acquired via experience. It is important to note that the PCCs who volunteered to participate in this study may be clinicians who have thought deeply about chronic pain. Other PCCs who have limited experience prescribing opioids may have little knowledge of the negative effects beyond addiction and misuse. There is a need for a shared clinical framework for conceptualizing the management of chronic pain and the deployment of LTOT and its consequences. Less-experienced PCCs may benefit from practical explanations of the impact of opioid therapy on function, as well as strategies experienced PCCs have developed for managing chronic pain.

Notably, the Centers for Disease Control and Prevention and the Food and Drug Administration provide evidencebased resources that are universally available. We recommend that primary care organizations explore strategies for integrating these materials into staff meetings and continuing education so that PCCs have an opportunity to discuss research findings and recommendations with colleagues and clinic leadership. Moving research findings into clinic practice via organizational initiatives and clinical cognitive support may increase the likelihood that clinicians will appropriately leverage findings in practice. Even some of the PCCs in this study who have a strong interest in chronic pain reported difficulty finding the time to learn about and integrate the latest research without organizational support. Ready access to research findings that are directly relevant to assessment and treatment planning may help place personal observations in a larger context and guide decision-making. One promising approach is the introduction of Special Care Access Network Extension for Community Healthcare Outcomes (SCANECHO) programs by the Veterans Health Administration to help educate PCCs about effective chronic pain management.

A second implication of these findings is that creating organizational change to better accommodate the needs of patients with chronic pain may be beneficial. In many ways, treating pain is a problem of time. It takes time to establish rapport and trust, elicit information about pain, and manage patient expectations. One common strategy to manage time is to delegate tasks, leveraging support staff such as licensed practical nurses. For chronic pain, this has limited utility and, in fact, may be a barrier to the much-needed rapport and trust. Thus, in spite of the many barriers, we recommend exploration of strategies to increase the time available for PCCs to interact with patients with chronic pain.

A third implication is that time is ripe for innovations to better support PCCs in managing patients with chronic noncancer pain. Clinical cognitive support applications that provide context-specific recommendations and resources tailored to specific patients are recommended. For example, one promising approach is to provide visualizations that aid PCCs in quickly reviewing current and historical treatment plans, as they are reminded of applicable, evidence-based treatment options. (27)

There are important limitations to this study. First, PCCs who volunteered to participate in this study are likely those with an interest in chronic pain and thus may be more knowledgeable on this topic than others. Second, participants were recruited from a single region of the USA that has been hit particularly hard by the opioid crisis at a time when opioid prescribing and opioid deaths were prominent in the news cycle. This likely has influenced beliefs and practice in ways that may be different from other regions, and may have steered our interviewees toward the challenges associated with opioid prescribing over other relevant pain management topics. Third, our analysis focused primarily on challenging cases; therefore, cases in which patients did well with opioid therapy are under-represented in this discussion. Fourth, some PCCs participated in more interviews than others and thus had more opportunity to provide examples and insights. 


\section{CONCLUSIONS}

This paper highlights how PCCs' experiences inform their beliefs about LTOT and guide their evolving strategies for assessing and treating patients with chronic noncancer pain. PCC beliefs about opioid therapy generally align with the research base but may have some important gaps. In the context of the opioid epidemic in the USA, many PCCs are actively seeking information and developing hypotheses about the effectiveness of opioids, as well as the dangers. Furthermore, many report that the strategies they have developed to motivate patients with chronic pain require time that is difficult to find in the typical U.S.-based primary care clinic.

This creates fertile ground for interventions that are not pharmacological, but rather focus on (1) strategies to make research findings readily accessible, (2) mechanisms to support those experienced with managing patients with chronic pain in sharing knowledge and effective strategies they have developed, and (3) developing models of care that support PCCs in spending time with patients to develop rapport and trust, elicit information about pain, and manage patient expectations.

Although the findings reported here represent beliefs and strategies in the context of the opioid crisis in the USA, the implications of these findings are likely to be relevant beyond this time and context. PCCs will continue to provide care for patients with chronic pain long after the current opioid crisis is over.

Acknowledgments: We thank Burke Mamlin for his contributions to this research.

Corresponding Author: Laura G. Militello, MA; Applied Decision Science, LLC, 5335 Far Hills Ave, Suite 103, Dayton, OH 45429, USA (e-mail: l.militello@applieddecisionscience.com).

Funding This project was supported by grant number R01HSO23306 from the Agency for Healthcare Research and Quality.

\section{Compliance with Ethical Standards:}

All study procedures were approved by the Indiana University Institutional Review Board.

Conflict of Interest: All the authors on this manuscript received funding from an $A H R Q$ grant during the conduct of this study. Outside of the submitted work, Dr. Hurley reports grants and personal fees from Medtronic and grants from Boston Sci; Dr. Anders reports grants from $\mathrm{NIH}$; and Dr. Harle reports personal fees from Indiana Health Information Exchange, New York eHealth Collaborative, and RTI International. In addition, Dr. Harle has a patent pending: Methods and systems for risk assessment and risk prediction opioid prescription and pain management treatment.

Disclaimer: The content is solely the responsibility of the authors and does not necessarily represent the official views of the Agency for Healthcare Research and Quality.

\section{REFERENCES}

1. Klimas J, Gorfinkel L, Fairbairn N, Amato L, Ahamad K, Nolan S, et al. Strategies to identify patient risks of prescription opioid addiction when initiating opioids for pain: a systematic review. JAMA Netw Open 2019;2(5):e193365-e193365.

2. Lagisetty PA, Healy N, Garpestad C, Jannausch M, Tipirneni R, Bohnert ASB. Access to primary care clinics for patients with chronic pain receiving opioids. JAMA Netw Open 2019;2(7):e196928-e196928.

3. Kroenke K, Alford DP, Argoff C, Canlas B, Covington E, Frank JW, et al. Challenges with implementing the centers for disease control and prevention opioid guideline: a consensus panel report. Pain Med. 2019;20(4):724-35.

4. Kennedy LC, Binswanger IA, Mueller SR, Levy C, Matlock DD, Calcaterra SL, et al. "Those conversations in my experience don't go well": A qualitative study of primary care provider experiences tapering long-term opioid medications. Pain Med. 2018;19(11):2201-11.

5. Kenny DT. Constructions of chronic pain in doctor-patient relationships: bridging the communication chasm. Patient Educ Couns. 2004;52(3):297-305.

6. Crowley-Matoka M. True G. No one wants to be the candy man: ambivalent medicalization and clinician subjectivity in pain management. Cult Anthropol. 2012;27(4):689-712.

7. Jamison RN, Sheehan KA, Elizabeth Scanlan NP, Ross EL. Beliefs and attitudes about opioid prescribing and chronic pain management: survey of primary care providers. J Opioid Manag. 2014;10(6):375-82.

8. Upshur CC, Luckmann RS, Savageau JA. Primary care provider concerns about management of chronic pain in community clinic populations. J Gen Intern Med. 2006;21(6):652-5.

9. Centers for Disease Control and Prevention. Guideline for prescribing opioids for chronic pain. J Pain Palliat Care Pharmacother. 2016; 30(2):138-7.

10. Given LM. 100 questions (and answers) about qualitative research. SAGE Publications; Thousand Oaks 2015.

11. Saunders B, Sim J, Kingstone T, Baker S, Waterfield J, Bartlam B, et al. Saturation in qualitative research: exploring its conceptualization and operationalization. Qual Quant. 2018;52(4):1893-907.

12. Urquhart C. Grounded theory for qualitative research: A practical guide. Sage; 2012.

13. Crandall BW. A comparative study of think-aloud and critical decision knowledge elicitation methods. ACM SIGART Bull. 1989;(108):144-6.

14. Crandall B, Klein G, Hoffman RR. Working minds: A practitioner's guide to cognitive task analysis. Cambridge: Mit Press; 2006.

15. Nowell LS, Norris JM, White DE, Moules NJ. Thematic analysis: Striving to meet the trustworthiness criteria. Int $\mathrm{J}$ Qual Methods. 2017;16(1): 1609406917733847.

16. Frank JW, Lovejoy TI, Becker WC, Morasco BJ, Koenig CJ, Hoffecker $\mathbf{L}$, et al. Patient outcomes in dose reduction or discontinuation of longterm opioid therapy: a systematic review. Ann Intern Med. 2017;167(3):181-91.

17. Krebs EE, Gravely A, Nugent S, Jensen AC, DeRonne B, Goldsmith ES, et al. Effect of opioid vs nonopioid medications on pain-related function in patients with chronic back pain or hip or knee osteoarthritis pain: the SPACE randomized clinical trial. Jama. 2018;319(9):872-82.

18. Hoffman EM, Watson JC, St Sauver J, Staff NP, Klein CJ. Association of long-term opioid therapy with functional status, adverse outcomes, and mortality among patients with polyneuropathy. JAMA Neurol. 2017;74(7):773-9.

19. Berna C, Kulich RJ, Rathmell JP. Tapering long-term opioid therapy in chronic noncancer pain: evidence and recommendations for everyday practice. In: Mayo Clinic Proceedings. Elsevier; 2015. p. 828-42.

20. Marion Lee M, Sanford Silverman M, Hans Hansen M, Vikram Patel M, Laxmaiah Manchikanti MD. A comprehensive review of opioidinduced hyperalgesia. Pain Physician. 2011;14:145-61.

21. Oliva EM, Bowe T, Tavakoli S, Martins S, Lewis ET, Paik M, et al Development and applications of the Veterans Health Administration's Stratification Tool for Opioid Risk Mitigation (STORM) to improve opioid safety and prevent overdose and suicide. Psychol Serv. 2017;14(1):34.

22. Ballantyne JC, Sullivan MD, Koob GF. Refractory dependence on opioid analgesics. Pain. 2019;160(12):2655-60.

23. Manhapra A, Arias AJ, Ballantyne JC. The conundrum of opioid tapering in long-term opioid therapy for chronic pain: a commentary. Subst Abus. 2018;39(2):152-61. 
24. Driscoll MA, Knobf MT, Higgins DM, Heapy A, Lee A, Haskell S. Patient experiences navigating chronic pain management in an integrated health care system: A qualitative investigation of women and men. Pain Med. 2018;19(suppl_1):S19-29.

25. Østbye T, Yarnall KSH, Krause KM, Pollak KI, Gradison M, Michener JL. Is there time for management of patients with chronic diseases in primary care? Ann Fam Med. 2005;3(3):209-14.

26. Konrad TR, Link CL, Shackelton RJ, Marceau LD, von Dem Knesebeck O, Siegrist J, et al. It's about time: physicians' perceptions of time constraints in primary care medical practice in three national healthcare systems. Med Care. 2010;48(2):95.

27. Harle CA, Dilulio J, Downs SM, Danielson EC, Anders S, Cook RL, et al. Decision-Centered Design of Patient Information Visualizations to Support Chronic Pain Care. Appl Clin Inform. 2019;10(04):719-28.

Publisher's Note Springer Nature remains neutral with regard to jurisdictional claims in published maps and institutional affiliations. 